\title{
Dynamically-generated baryon resonances with heavy flavor
}

\author{
Olena Romanets* ${ }^{* \dagger}$ \\ KVI, University of Groningen, Zernikelaan 25, 9747 AA Groningen, The Netherlands \\ E-mail: olena.romanets@gmail.com
}

Carmen García-Recio and Lorenzo Luis Salcedo

Departamento de Física Atómica, Molecular y Nuclear, and Instituto Carlos I de Física Teórica

y Computacional, Universidad de Granada, Granada, E-18071, Spain

\section{Juan Nieves}

Instituto de Física Corpuscular (centro mixto CSIC-UV), Institutos de Investigación de Paterna, Aptdo. 22085, Valencia, 46071, Spain

\section{Laura Tolos}

Institut de Ciències de l'Espai (IEEC/CSIC), Campus Universitat Autònoma de Barcelona, Facultat de Ciències, Torre C5, Bellaterra, E-08193, Spain

\begin{abstract}
We study baryon resonances with heavy flavor in a molecular approach, thus as dynamically generated by meson-baryon scattering. This is accomplished by using a unitary coupled-channel model taking, as bare interaction, the extension to four flavors and spin symmetry of the WeinbergTomozawa interaction potential. A special attention is paid to the inclusion of heavy-quark spin symmetry and to the studies of the generated baryon resonances which complete the heavy-quark spin multiplets. We reproduce few charmed and strange baryon resonances found experimentally, that is, $\Lambda_{c}(2595), \Lambda_{c}(2625), \Xi_{c}(2790), \Xi_{c}(2815)$, and make predictions for more states. The bottom-flavored $\Lambda_{b}(5912)$ and $\Lambda_{b}(5920)$ states, found by the LHCb collaboration, are also obtained by our model, thus these resonances can be interpreted as molecular states. We also study $\Xi_{b}$ resonances, which belong to the same SU(3) $\times$ HQSS multiplets as the observed $\Lambda_{b}$ particles. Finally we analyze hidden-charm baryon resonances. In this sector, we predict seven $N$-like and five $\Delta$-like states with masses around $4 \mathrm{GeV}$, most of them as bound states, and compare them with predictions from other models. The predicted states can be searched for in future experiments that involve studies of heavy-flavor physics, e.g. PANDA/FAIR, where charm physics will be analyzed.
\end{abstract}

XV International Conference on Hadron Spectroscopy

4-8/11/2013

Nara, Japan

\section{*Speaker.}

${ }^{\dagger}$ Supported by Spanish Ministerio de Economía y Competitividad (FIS2011-28853-C02-02, FIS2011-24149, FPA2010-16963), Junta de Andalucia (FQM-225), Generalitat Valenciana (PROMETEO/2009/0090) and EU HadronPhysics2 project (grant 227431). O. R. acknowledges support from the Rosalind Franklin Fellowship. L. T. acknowledges support from RyC Program, and FP7-PEOPLE-2011-CIG (PCIG09-GA-2011-291679). 


\section{Introduction}

The study of particles with heavy flavor is an active research topic, and among them baryon resonances have recently attracted a lot of attention. An interest in heavy-flavor physics in the last years was induced by the past and on-going experiments such as CLEO, Belle, BABAR, LHCb [1]. Moreover, the planned PANDA and CBM experiments at FAIR [2] will bring even more excitement in the field. It is an important task to understand the nature of possible new states, e.g. whether baryon resonances can be interpreted as three-quark states, or better as molecular states.

In this work we study baryon resonances with heavy (charm and bottom) flavor as molecular states, thus dynamically generated by the meson-baryon dynamics. There are several approaches used to study this type of states. The coupled-channels models turned out to be successful in describing some of the experimental data, and include unitarized coupled-channels models $[3,4$, 5, 6], the Jülich meson-exchange model [7] and schemes based on hidden gauge formalism [8]. In this work we review findings of the unitarized coupled-channels model based on the spin-flavor extension of the Weinberg-Tomozawa (WT) potential, that has been used for studying charmed strange and non-strange baryon resonances [9], hidden-charm baryon resonances [10], and bottom states [11].

\section{The phenomenological model}

We use an extension of the WT contact term interaction to SU(8) spin-flavor symmetry. The extended WT Hamiltonian for four flavors and three colors reads [12]

$$
H_{\mathrm{WT}}^{\mathrm{sf}}(x)=-\frac{\mathrm{i}}{4 f^{2}}:\left[\Phi, \partial_{0} \Phi\right]_{B}^{A} \mathscr{B}_{A C D}^{\dagger} \mathscr{B}^{B C D}:, \quad A, B, \ldots=1, \ldots, 8,
$$

where $\Phi_{B}^{A}(x)$ is the meson field, which contains the fields of $0^{-}$(pseudoscalar) and $1^{-}$(vector) mesons, and $\mathscr{B}^{A B C}$ is the baryon field, which is a completely symmetric tensor, containing the lowest-lying baryons with $J^{P}=\frac{1}{2}^{+}$and $\frac{3}{2}^{+}$. The Hamiltonian can be rewritten as

$$
H_{\mathrm{WT}}=H_{\mathrm{ex}}+H_{\mathrm{ac}},
$$

where $H_{\mathrm{ex}}$ is the exchange part, in which a quark is transferred from the meson to the baryon, and another one is transferred from the baryon to the meson, and $H_{\mathrm{ac}}$ is the annihilation-creation mechanism, where an antiquark in the meson annihilates with a similar quark in the baryon, with subsequent creation of a quark and an antiquark. It turns out [10] that the annihilation-creation part of the Hamiltonian can violate the heavy-quark spin symmetry (HQSS) when the annihilation or creation of $q \bar{q}$ pairs involves heavy flavor, since according to the HQSS the number of heavy quarks and the number of heavy antiquarks are separately conserved (this implies $\mathrm{U}_{c}(1) \times \mathrm{U}_{\bar{c}}(1)$ ). The HQSS group, which also includes a group of separate rotations of the $c$ quark and $\bar{c}$ antiquark, reads as $\mathrm{SU}_{c}(2) \times \mathrm{SU}_{\bar{c}}(2) \times \mathrm{U}_{c}(1) \times \mathrm{U}_{\bar{c}}(1)$. We modify the Hamiltonian in correspondence to the requirements of HQSS. Thus, in the sector of open-charm and open-bottom baryon resonances we eliminate possible coupled channels that contain heavy quark-heavy antiquark, since in this case the annihilation-creation mechanism would necessarily contain the heavy flavor. In the hidden-charm sectors, where in $H_{\mathrm{ac}}$ the annihilated and created antiquark is necessarily $\bar{c}$, the annihilation-creation 
part of the Hamiltonian is not present due to HQSS. [10]. After implementing this change into the Hamiltonian, the $\mathrm{SU}(8)$ symmetry is broken and the model possess $\mathrm{SU}(6) \times \mathrm{HQSS}$ symmetry, where $\mathrm{SU}(6)$ is the spin-flavor symmetry for the three light flavors.

We calculate the scattering amplitude by solving the on-shell Bethe-Salpeter equation (BSE) in coupled channels. The meson-baryon propagators that appear in the BSE are regularized using the subtraction point method (see [9] for details). Baryon resonances appear as poles of the scattering amplitude on the complex energy plane. Poles on the first Riemann sheet that appear on the real axis below threshold are bound states, and the ones in the second Riemann sheet below the real axis and above threshold are identified with resonances. We find the mass and the width as well as the couplings to the meson-baryon channels of a baryon resonance from the behavior of the scattering amplitude around the pole, that is, the coordinate of the pole in the complex energy plane and the residue, respectively.

The $\mathrm{SU}(6) \times$ HQSS symmetry is broken in nature, thus, we break it adiabatically to the $\mathrm{SU}(2)$ isospin symmetry, by implementing the physical hadron masses and meson decay constants [9]. In this way we could follow the evolution of the poles while breaking the symmetry and classify the found states under the corresponding $\mathrm{SU}(6), \mathrm{SU}(3)$, and HQSS group multiplets.

\section{Results for dynamically-generated baryon resonances}

\subsection{Open-charm baryon resonances}

With the described model a number of baryon resonances have been studied. Let us present our results for the charmed baryon resonances with charm $C=1$. Such states will be studied in the upcoming PANDA experiment at FAIR [2].

We have studied non-strange baryon resonances with $C=1$ and isospin 0 , these are $\Lambda_{c}$ states. We find the experimentally known $\Lambda_{c}(2595)$ resonance. In our model it appears around $2618.8-$ $i 0.6 \mathrm{MeV}$, and has a possible decay channel $\Sigma_{c} \pi$. The experimental value of the width of $\Lambda_{c}(2595)$ $3.6_{-1.3}^{+2.0} \mathrm{MeV}$ is not reproduced, since we have not included the three-body decay channel $\Lambda_{c} \pi \pi$, which represents about $67 \%$ of the decay events [13]. We also find another $J=1 / 2$ baryon resonance with a very close mass of $2617.3 \mathrm{MeV}$, but with a big width of about $90 \mathrm{MeV}$. This resonance together with $\Lambda_{c}(2595)$ makes a two-pole structure, similar to the case of $\Lambda(1405)$. Moreover, this resonance forms a HQSS doublet with the pole at $2666.6-i 27 \mathrm{MeV}$, which we identify with the $\Lambda_{c}(2625) J=3 / 2$ resonance, coupling strongly to the $\Sigma_{c}^{*} \pi$ channel. The experimental value of the width $(\Gamma<1.9 \mathrm{MeV})$ is not reproduced, but if we lower the mass of the found resonance to $2625 \mathrm{MeV}$ by slightly changing the subtraction point, the width will decrease significantly. There is another predicted $\Lambda_{c}$ resonance, with the mass $2828.4 \mathrm{MeV}$ and a small width.

We predict the existence of three $J=1 / 2 \Sigma_{c}$ resonances with masses 2571.5, 2622.7, and 2643.4 MeV. The first one has a narrow width, and the other two are broad $(\Gamma=188$ and $87 \mathrm{MeV}$, respectively). Besides, our model predicts two $J=3 / 2 \Sigma_{c}$ states, a bound state at $2568.4 \mathrm{MeV}$, and a broad $(\Gamma=67 \mathrm{MeV})$ resonance at $2692.9 \mathrm{MeV}$. The verification of these predictions requires more experimental data.

Further, we find nine $\Xi_{c}$ baryon resonances. One of them we identify with the experimental $\Xi_{c}(2790)(J=1 / 2)$ resonance with the decay channel $\Xi_{c}^{\prime} \pi$, and another one with the experimental 
$\Xi_{c}(2815)(J=3 / 2)$, with the decay channel $\Xi_{c}^{*} \pi$. The predicted features of the other seven $\Xi_{c}$ resonances can be found in Ref. [9]. We also predict the existence of five $\Omega_{c}$ states, all of them as bound states, three with $J=1 / 2$, and two with $J=3 / 2$. The masses of these states are predicted to lie between 2800 and $3000 \mathrm{MeV}$.

\subsection{Hidden-charm baryon resonances}

We have studied $N$-like and $\Delta$-like baryon resonances with hidden charm, thus containing $c \bar{c}$. We find seven $N$-like states, five of them as bound states, and two of them with the small widths $(0.1$ and $2.8 \mathrm{MeV}$ ), with masses between 3900 and $4050 \mathrm{MeV}$. Besides, we predict five $\Delta$-like hidden-charm particles, all of them as bound states, with masses between 4000 and $4310 \mathrm{MeV}$. The two heaviest ones, with masses 4306.2 and $4306.8 \mathrm{MeV}$, lie very close to the $\Delta J / \psi$ threshold (4306.9 MeV), and therefore they appear as cusps. The general pattern of the hidden-charm states is that they couple weakly to $N \eta_{c}$ and $N J / \psi$ channels, and have significantly bigger couplings to the channels that contain $\Lambda_{c}, \Sigma_{c}$ or $\Sigma_{c}^{*}$ baryon, and $\bar{D}$ or $\bar{D}^{*}$ meson.

The states in this sector form HQSS multiplets (not only doublets as it is in the open-charm sector). Many of the states differ from each other by the way the spin of the heavy quark-antiquark pair couples to the light-quarks content. Because HQSS implies that the interaction dependent on the spin state of the heavy quark vanishes in the infinite quark limit, such states are degenerate under HQSS.

The $N$-like crypto-exotic resonances were previously predicted by the zero-range vector exchange model [6]. The $N$ resonances studied in Ref. [6] are about $500 \mathrm{MeV}$ lighter than those found in our model. The hidden-gauge formalism predicts the masses of hidden-charm $N$ to be about 400 $\mathrm{MeV}$ larger $[14,15]$. It has been shown that this difference is mainly due to a different renormalization scheme [15]. The masses of $N$-like states predicted by our model lie close to the ones predicted by the constituent quark model of Ref. [16] using the hyperfine chiral interaction based on meson exchange, whereas the instanton-induced and color-magnetic hyperfine interactions produce higher masses for the resonances [16].

\subsection{Baryon resonances with bottom}

Our model reproduces the $\Lambda_{b}(5912)$ and $\Lambda_{b}(5920)$ resonances, which were discovered by the LHCb collaboration in the $\Lambda_{b} \pi \pi$ spectrum [17]. In our scheme these states form a HQSS doublet, which explains the closeness of their masses. We find $\Lambda_{b}(5912)$ and $\Lambda_{b}(5920)$ as bound states, as we do not consider three-body decay channels. Such decay can be realized through the intermediate decay to $\Sigma_{b} \pi$ [ $\Sigma_{b}^{*} \pi$ for $\left.\Lambda_{b}(5920)\right]$, with consequential decay of the virtual $\Sigma_{b}\left(\Sigma_{b}^{*}\right)$ to $\Lambda_{b} \pi$.

We also find a $\Lambda_{b}$ bound state with a mass $5797.6 \mathrm{MeV}$, without any possible strong decay, and a bound state with a mass of $6009.3 \mathrm{MeV}$ that decays to $\Sigma_{b} \pi$. Apart from studying $\Lambda_{b}$ resonances, we have also made predictions for $\Xi_{b}$ resonances, which belong to the same SU(3) $\times$ HQSS irreps as $\Lambda_{b}(5912)$ and $\Lambda_{b}(5920)$. We find three spin- $1 / 2 \Xi_{b}$ states, with zero or small widths and masses 5874., 6035.4 , and $6072.8 \mathrm{MeV}$, and one spin-3/2 $\Xi_{b}$ bound state with a mass of $6043.3 \mathrm{MeV}$.

\section{Summary}

A number of heavy-flavored baryon resonances, namely charmed baryon resonances, hidden- 
charm states, and baryon resonances with bottom have been studied using a coupled-channels unitary approach. For this purpose we use a spin-flavor extension of the WT interaction to four flavors implementing HQSS constraints. We have obtained a number of $C=1$ baryon resonances, among

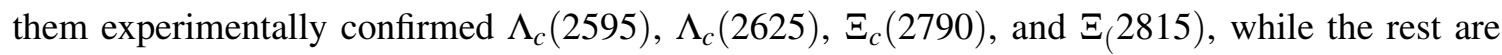
predictions of the model. We have also studied hidden-charm $N$ and $\Delta$-like states, and compared them with the predictions of other theoretical models. New experimental data is expected in the future PANDA experiment at FAIR, which will help to constrain our model as well as verify our predictions. Finally, baryon resonances with bottom have been studied. Our model generates experimental $\Lambda_{b}(5912)$ and $\Lambda_{b}(5920)$ states as molecular states, and we have predicted the existence of other two $\Lambda_{b}$ resonances and few $\Xi_{b}$ states.

\section{References}

[1] www.lepp.cornell.edu/Research/EPP/CLEO, belle.kek.jp, www-public.slac.stanford.edu/babar, http://lhcb-public.web.cern.ch/lhcb-public/.

[2] http://www.fair-center.eu/.

[3] M. F. M. Lutz and E. E. Kolomeitsev, Nucl. Phys. A 730, 110 (2004); M. F. M. Lutz and E. E. Kolomeitsev, Nucl. Phys. A 755, 29 (2005).

[4] L. Tolos, J. Schaffner-Bielich and A. Mishra, Phys. Rev. C 70, 025203 (2004); T. Mizutani and A. Ramos, Phys. Rev. C 74, 065201 (2006).

[5] C. E. Jimenez-Tejero, A. Ramos and I. Vidana, Phys. Rev. C 80, 055206 (2009).

[6] J. Hofmann and M. F. M. Lutz, Nucl. Phys. A 763, 90 (2005); Nucl. Phys. A 776, 17 (2006).

[7] J. Haidenbauer et al., Eur. Phys. J. A 33, 107 (2007); J. Haidenbauer et al., Eur. Phys. J. A 37, 55 (2008).

[8] J. -J. Wu et al. Phys. Rev. Lett. 105, 232001 (2010); K. P. Khemchandani et al., Phys. Rev. D 83, 114041 (2011).

[9] O. Romanets, L. Tolos, C. Garcia-Recio, J. Nieves, L. L. Salcedo, and R.G.E. Timmermans, Phys. Rev. D 85, 114032 (2012).

[10] C. Garcia-Recio, J. Nieves, O. Romanets, L. L. Salcedo, and L. Tolos, Phys. Rev. D 87, 074034 (2013).

[11] C. Garcia-Recio, J. Nieves, O. Romanets, L. L. Salcedo, and L. Tolos, Phys. Rev. D 87, 034032 (2013).

[12] C. Garcia-Recio, J. Nieves and L. L. Salcedo, Phys. Rev. D 74, 036004 (2006).

[13] J. Beringer et al. (Particle Data Group), Phys. Rev. D 86, 010001 (2012).

[14] J. -J. Wu, R.et al., Phys. Rev. C 84, 015202 (2011); J. -J. Wu et al., Phys. Rev. C 85, 044002 (2012).

[15] C. W. Xiao, J. Nieves, E. Oset, Phys. Rev. D 88056012 (2013).

[16] S. G. Yuan et al. Eur. Phys. J. A 48,61 (2012).

[17] R. Aaij et al. [LHCb Collaboration], Phys. Rev. Lett. 109 (2012) 172003. 